\title{
Erratum to: Plaque Rupture and Thrombosis: the Value of the Atherosclerotic Rabbit Model in Defining the Mechanism
}

\author{
Oliver G. Abela ${ }^{1}$. Chowdhury H. Ahsan ${ }^{1}$. Fadi Alreefi ${ }^{2} \cdot$ Negar Salehi $^{3} \cdot$ Imran Baig $^{2}$. \\ Abed Janoudi $^{2}$ - George S. Abela ${ }^{2,4,5}$
}

Published online: 28 June 2016

(C) Springer Science+Business Media New York 2016

Erratum to: Curr Atheroscler Rep (2016)

DOI 10.1007/s11883-016-0587-0

The reference of permissions granted for Figure 2 is mistakenly listed as Ref. 52, when the correct reference is Ref. 51, for "Pham T, Hua N, Phinikaridou A, Killiany R, Hamilton J. Early in vivo discrimination of vulnerable atherosclerotic plaques that disrupt: a serial MRI study. Atherosclerosis. 2016;244:101-7."

The online version of the original article can be found at http://dx.doi. org/10.1007/s11883-016-0587-0.

\footnotetext{
George S. Abela

george.abela@ht.msu.edu

1 Department of Medicine, Division of Cardiovascular Medicine, University of Nevada, Las Vegas, NV, USA

2 Division of Cardiovascular Medicine, Michigan State University, East Lansing, MI, USA

3 Department of Medicine, Michigan State University, East Lansing, MI, USA

4 Department of Physiology, Division of Pathology, Michigan State University, East Lansing, MI, USA

5 Michigan State University, B208 Clinical Center, East Lansing, MI 48824, USA
} 